and had instead developed side effects to steroid therapy. Neither antilymphocyte globulin nor cyclosporin therapy have been attempted in view of the fact that the aplasia is of long standing.

Pajor $e t a{ }^{\beta}$ reported no relationship between pregnancy and subsequent outcome of aplastic anaemia but all pregnancies in their four patients with active aplastic anaemia were terminated early. It would appear that in our patient, pregnancy may have contributed to the worsening of her aplastic anaemia. Postpartum stress may also have caused an increase in symptoms.

1 Camitta BM, Storb R, Thomas ED. Aplastic anaemia: pathogenesis, diagnosis, treatment and prognosis. $N$ Engl $\mathcal{F}$ Med 1982; 306: 645-52, 712-8.

2 Bacigalupo A, Hows J, Gluckman E, et al. Bone marrow transplantation versus immunosuppression for the treatment of severe aplastic anaemia: a report of the EBMT SAA working party. $\mathrm{Br}$ f Haematol 1988; 70: 177-82.

3 Pajor A, Kelemen E, Szakacs Z, Lehoczky D. Pregnancy in idiopathic aplastic anaemia (report of 10 patients). Eur $\mathcal{F}$ Obstet Gynecol Reprod Biol 1992; 45: 19-25.

4 Suda T, Omine M, Tuchiya J, Maekawa T. Prognostic aspects of aplastic anaemia. Experience on six cases and aspects of aplastic anaemia. Experience on six
review of the literature. Blut 1978; 36: 285-98.

\section{Conclusion}

Pregnancy following a diagnosis of idiopathic aplastic anaemia is not advisable due to the risk of infection and bleeding and platelet refractoriness. Contraception and termination of early pregnancy should continue to be recommended. In the event of a presentation during late pregnancy, as in this patient, recent advances in supportive care and transfusion medicine may, however, lead to a successful outcome.

5 Aitchison RG, Marsh JC, Hows JM, Russel NH, Gordon S. Pregnancy associated with aplastic anaemia: a report of five cases and review of current management. $\mathrm{Br} \mathcal{F}$ Haematol 1989; 73: 541-5.

6 McFarland JG, Anderson AJ, Slichter SJ. Factors influencing the transfusion response to HLA-selected apheresis donor platelets in patients refractory to random platelet concentrates. Br $\mathcal{F}$ Haematol 1989; 73: 380-6.

\title{
Systemic lupus erythematosus presenting as effuso-constrictive pericarditis
}

\author{
SR McMechan, BM McClements, PP McKeown, SW Webb, AAJ Adgey
}

\begin{abstract}
Summary
We describe a 62-year-old woman in whom systemic lupus erythematosus presented as life-threatening effuso-constrictive pericarditis. Surgical drainage of the pericardium was required and the patient made a satisfactory recovery. At six-months follow-up, while taking hydroxychloroquine and a non-steroidal anti-inflammatory agent, she remains well.
\end{abstract}

Keywords: systemic lupus erythematosus, pericarditis

\section{Case report}

A 62-year-old woman was admitted with a six-day history of flu-like symptoms and pleuritic-type central chest discomfort. Past history included goitre and a septicaemic illness several years ago. One year prior to this admission she had suffered an episode of dizziness and tinnitus, thought to be a cerebral transient ischaemia attack, for which she was taking aspirin. She had required antidepressant drug therapy for the past four years following the death of her husband.

On examination she appeared gravely ill, apyrexic, cold and clammy with poorly perfused peripheries. Her heart rate was 130 beats/ min (sinus tachycardia) and her systolic blood pressure was $90 \mathrm{mmHg}$. Jugular venous pressure was elevated. Two heart sounds were normal and examination of chest and abdomen was normal. Chest X-ray revealed cardiomegaly. The ECG showed reduced voltages with ST segment elevation in the anterior and lateral leads (figure 1). Trans-thoracic echocardiography showed a $1.5 \mathrm{~cm}$ pericardial effusion. The working diagnosis was extensive acute anterolateral myocardial infarction with subacute rupture.

Emergency cardiac catheterisation was carried out in order to confirm this diagnosis. Surprisingly, the coronary arteries were normal and there were no regional wall movement

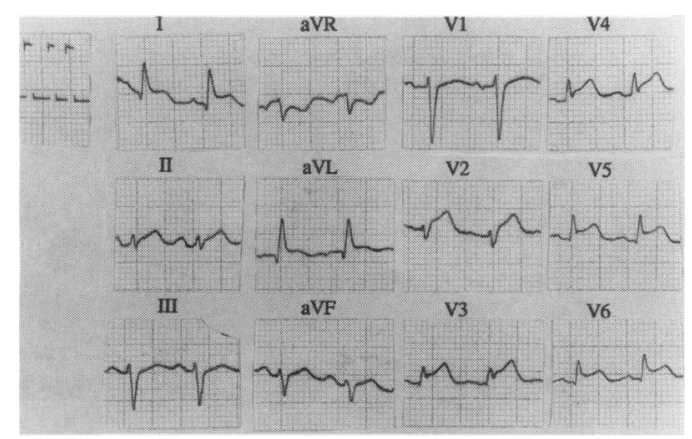

Figure 1 Electrocardiogram demonstrating ST segment elevation in leads I, II, aVL, and V2-V6 
defects to suggest myocardial infarction. There was moderate global impairment of left ventricular function. There was elevation and equalisation of the diastolic pressures in all four cardiac chambers, in keeping with constriction. There was a prominent y descent in the right atrial pressure waveform (figure 2), reflecting rapid unimpeded early diastolic filling, which is abruptly halted when the intracardiac volume is limited by the non-compliant pericardium.

Trans-oesophageal echocardiography confirmed the $1-2 \mathrm{~cm}$ pericardial effusion with diastolic collapse of the right atrium (figure 3 ). In addition, some increase in echogenicity was noted around the superior vena cava with the suspicion of extrinsic compression of the right ventricle. Computed tomography (CT) scan did not show any mass lesion within the mediastinum but did confirm thickening of the anterior pericardium over the right ventricle.

Pericardial aspiration produced $250 \mathrm{ml}$ of a straw-coloured exudate, containing polymorphs and reactive mesothelial cells. There were no mycobacteria. A coagulase-negative staphylococcus was isolated from the aspirate on the third day and was thought to be a contaminant. Nevertheless the patient was commenced on ceftazidime and flucloxacillin.

The patient failed to improve with inotropic agents and remained in a low cardiac output state with a systolic blood pressure of $80 \mathrm{mmHg}$ and poor peripheral perfusion. Pericardial exploration was undertaken on the third day and at operation the pericardial sac was noted to be very tense. On opening the pericardium, $300 \mathrm{ml}$ of straw-coloured fluid were released under pressure with immediate improvement in blood pressure to 120 /

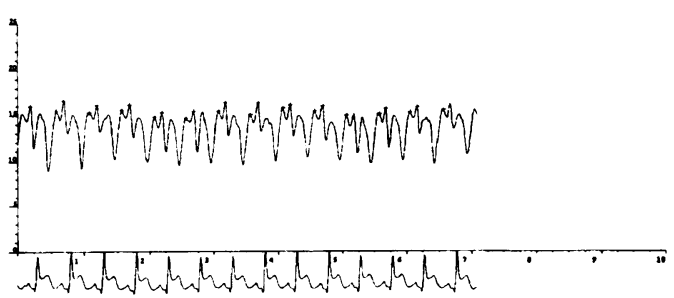

Figure 2 Right atrial (RA) pressure waveform indicating mean RA pressure of $13-14 \mathrm{mmHg}$ and prominent y descent

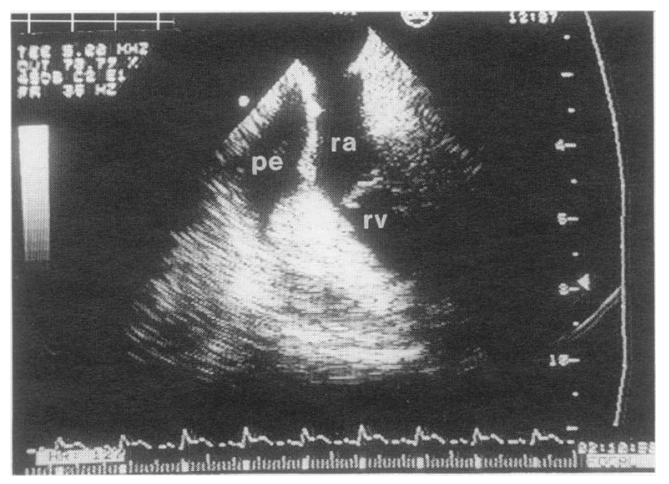

Figure 3 Trans-oesophageal echocardiogram; ra = right atrium, $r v=$ right ventricle, $p e=$ pericardial effusion
$82 \mathrm{mmHg}$. The pericardium was moderately thickened and the myocardium was oedematous. The pericardium was left open with a pericardial drain in situ. Pericardial biopsy revealed fibrosis without evidence of granulomata or malignancy and two weeks later biopsy of the right ventricle via the transvenous route also showed fibrosis.

Screening for a wide range of infective agents was negative. Erythrocyte sedimentation rate (ESR) and C-reactive protein were normal Immunological studies showed positive antinuclear antibody (IgG 20, IgM 320), antibody to neutrophil cytoplasmic antigen (pANCA) and low C4 component of complement $(0.18 \mathrm{~g} / 1$, normal $0.2-0.5 \mathrm{~g} / \mathrm{l}$ ). Total haemolytic complement and antibody to double-stranded DNA were normal. Rheumatoid factor and antibodies to nuclear and related antigens were negative.

Four weeks after the initial presentation she developed a low-grade fever together with pleuritic pain in the region of the left base accompanied by a pleural rub and an effusion. The ESR and C-reactive protein rose to $100 \mathrm{~mm}$ in the first hour and $308 \mathrm{mg} / \mathrm{l}$, respectively. Repeat echocardiogram showed only a small rim of pericardial fluid and a ventilation/ perfusion lung scan showed no evidence of pulmonary embolism.

A diagnosis of systemic lupus erythematosus was suspected on the basis of pericarditis, pleurisy, positive antinuclear antibody, low C4 component of complement and evidence of a depressive illness in the past. There was no proteinuria or skin rash. A lupus band test revealed the presence of fine granules of IgM and Civatte bodies along the epidermaldermal junction.

The patient's condition improved with rest and non-steroidal anti-inflammatory drugs. After ophthalmological assessment she was commenced on prophylactic hydroxychloroquine. At review 2, 4 and 6 months later, she was feeling well and ESR and C-reactive protein had returned to normal.

\section{Discussion}

In this patient, the diagnosis of systemic lupus erythematosus is supported by the finding of serositis (pericarditis and pleurisy), a history of depressive illness and a significant titre of $N$ antinuclear antibody on repeated occasions. Although the revised criteria of the American Rheumatism Association (after Tan et $a l^{1}$ ) require four of 11 criteria for the diagnosis of lupus, it is possible to be confident of the diagnosis on less strict grounds. ${ }^{2}$

The cardiac manifestations of systemic lupus erythematosus are multiple with pericardial disease being the most common. While pericardial effusion is detectable by echocardiography in $27-42 \%{ }^{3,4}$ of patients it is rarely haemodynamically significant. In a series of 395 lupus patients, $75(19 \%)$ had pericarditis but only 10 $(2.5 \%)$ had cardiac tamponade. ${ }^{5}$ The occurrence of subsequent constrictive pericarditis is even less frequent with only occasional reports in the literature. ${ }^{6}$ It is particularly notable in 
this case that constrictive pericarditis was the presenting feature of the disease and that it appears to have been of rapid onset.

The mode of presentation in our patient was particularly interesting. The initial diagnosis was acute anterolateral myocardial infarction with subacute rupture. The transthoracic echocardiogram was a technically difficult bedside study in a gravely ill patient. It revealed a pericardial effusion but the right-sided structures were difficult to visualize and at that stage constrictive pericarditis was not suspected. The diagnosis of constrictive pericarditis by echocardiography can be difficult even in optimal conditions and when the operator is specifically looking for the signs of this condition. In this patient the diagnosis was made by cardiac catheterisation which revealed the characteristic haemodynamic changes of constriction.

The aetiology of pericarditis in systemic lupus erythematosus may be active disease, uraemia or infection. In this case the initial presentation was complicated by the identification of a coagulase-negative Staphylococcus isolated from pericardial aspirate. Coagulasenegative Staphylococcus is unlikely to be the causative agent in a non-immunocompromised host. Systemic lupus erythematosus is associated with some suppression of the immune response and although this patient demonstrated low C4 complement, she had a normal leucocyte response, was not on corticosteroid

1 Tan EM, Cohen AS, Fries JF, et al. The 1982 revised criteria for the classification of systemic lupus erythematosus. Arthritis Rheum 1982; 25: 1271-7.

2 Mills JA. Systemic lupus erythematosus. N Engl ₹ Med 1994, 330: 1871-9.

3 Cervera R, Font J, Pare C, et al. Cardiac disease in systemic lupus erythematosus: prospective study of 70 patients. Ann Rheum Dis 1992; 51: 156-9.

4 Doherty NE, Feldman G, Maurer G, Siegel RJ. Echocardiographic findings in systemic lupus erythematosus. $A m \mathcal{f}$ Cardiol 1988; 61: 1144 . treatment, and did not appear to be susceptible to any other opportunistic infective agents.

Renal involvement is common in lupus patients with pericarditis, occurring in six of a series of 10 patients with tamponade. ${ }^{5}$ This association may reflect active multisystem involvement or the presence of severe nephritis with uraemia and consequent pericardial effusion. This patient had no renal impairment, proteinuria, or oedema, thus making the pericarditis more likely to be the result of the active lupus process.

At operation the myocardium was oedematous and a later right ventricular biopsy showed increased fibrosis. In the absence of coronary artery disease, it is probable that this is also a manifestation of lupus involvement.

In general, high dose corticosteroids are indicated in severe pericardial disease due to systemic lupus erythematosus. ${ }^{5}$ In this case the identification of coagulase-negative Staphylococcus was a contraindication to immunosuppressive therapy and the diagnosis of lupus was not confirmed until the convalescent period. Pericardial drainage was an adequate measure without the use of steroids. We elected to manage this patient with non-steroidal antiinflammatory drugs and the anti-malarial, hydroxychloroquine, rather than to institute long-term steroid treatment. It is interesting that in the few cases reported, low-dose steroids did not prevent the development of constrictive pericarditis. ${ }^{6}$

$5 \mathrm{Kahl} \mathrm{LE}$. The spectrum of pericardial tamponade in systemic lupus erythematosus: report of ten patients. Arthritis Rheum 1992; 35: 1343-9.

6 Jacobson EJ, Reza MJ. Constrictive pericarditis in systemic lupus erythematosus: demonstration of immunoglobulins in the pericardium. Arthritis Rheum 1978; 21: 972-4. 